\title{
Current research advances in microRNA-mediated regulation of Krüppel-like factor 4 in cancer: a narrative review
}

\author{
Xi Yang ${ }^{1,2 \#}$, Cheng Chen ${ }^{2 \#}, \mathrm{Li} \mathrm{Li}^{2}$, Tian Xiao ${ }^{2}$, Yong-Dong Zou ${ }^{2}$, Duo Zheng ${ }^{1,2}$ \\ ${ }^{1}$ National Center for International Research of Bio-targeting Theranostics, Guangxi Key Laboratory of Bio-targeting Theranostics, Collaborative \\ Innovation Center for Targeting Tumor Diagnosis and Therapy, Guangxi Talent Highland of Bio-targeting Theranostics, Guangxi Medical \\ University, Nanning, China; ${ }^{2}$ Shenzhen University International Cancer Center, Department of Cell Biology and Genetics, School of Medicine, \\ College of Life Sciences and Oceanography, Shenzhen University, Shenzhen, China \\ Contributions: (I) Conception and design: X Yang, C Chen; (II) Administrative support: D Zheng, YD Zou; (III) Provision of study materials or \\ patients: None; (IV) Collection and assembly of data: None; (V) Data analysis and interpretation: None; (VI) Manuscript writing: All authors; (VII) \\ Final approval of manuscript: All authors. \\ \#These authors contributed equally to this work. \\ Correspondence to: Duo Zheng. Shenzhen University International Cancer Center, Department of Cell Biology and Genetics, School of Medicine, A7- \\ 450, Shenzhen University, Lihu Campus, Nanshan District, Shenzhen 518055, China. Email: dzheng@szu.edu.cn; Yong-Dong Zou. College of Life \\ Sciences and Oceanography, A5-124, Shenzhen University, Lihu Campus, Nanshan District, Shenzhen 518055, China. Email: zouyd@szu.edu.cn.
}

Objective: The purpose of this study was to investigate the miRNAs and related mechanisms that regulates KLF4 in different cancers. Furthermore, we summarized the potential targets of miRNAs regulating the KLF4 pathway in cancer research.

Background: MiRNAs are single-stranded, endogenous non-coding small RNAs, some of which are related to human cancers. miRNAs carry out post-transcriptional gene regulation through translation inhibition and degradation of target messenger RNAs (mRNAs) via complementarily pairing with their 3' untranslated regions. KLF4 is an important transcription factor with complex involvement in cancer. Increasing evidence shows that miRNAs are dysregulated in cancer and can regulate cancer-related signaling pathways, thereby affecting tumor progression.

Methods: Systematic scientific literature searches were undertaken on PubMed using the following terms: "miRNAs and KLF4", "KLF4 and cancer", "miRNAs and cancer", and "miRNAs, KLF4 and cancer". Relevant papers were retrieved and further results were found by reviewing related papers and the references of the retrieved papers. We then conducted a narrative overview of the literature to summarize the results of the papers.

Conclusions: The role of KLF4 in cancer varies in a context-dependent manner. KLF4-regulating miRNAs in different tumors include miR-124, miR-9-5p, miR-10b, miR-18a, miR-25-3p, miR-10b, miR92a, miR-103, miR-155, miR-135b-5p, miR-32-5p, miR-148-3p, miR-152-3p, miR-10b, miR-25, miR3120-5p, miR-7, miR-1233-3p, miR-10b, miR-145, miR-139-5p, miR-16, miR-152, miR-375, and miR-145.

Keywords: MicroRNA (miRNA); Krüppel-like factor 4 (KLF4); regulation; cancer

Submitted Apr 20, 2021. Accepted for publication May 28, 2021.

doi: $10.21037 /$ atm-21-2347

View this article at: http://dx.doi.org/10.21037/atm-21-2347 


\section{Introduction}

MicroRNAs (miRNAs) are a family of naturally occurring, highly conserved non-coding single-stranded RNA molecules (approximately 19 to 25 nucleotides in length), which are present in various organisms and carry out a range of biological functions. By binding to specific complementary sites in the $3^{\prime}$ untranslated region (3' UTR) of target messenger RNAs (mRNAs), miRNAs regulate post-transcriptional gene expression by inducing translation inhibition and degradation of mRNAs, resulting in the silencing of target gene expression and affecting cell division, development, differentiation, and homeostasis $(1,2)$. Since miRNAs were first discovered in 1993, research into their functions has attracted much attention. Currently, miRNAs are known to be involved in the regulation of about $60 \%$ of coding genes and in the expression regulation of at least $30 \%$ of genes involved in biological processes such as cell proliferation, differentiation, and apoptosis $(3,4)$. Studies have found that miRNAs are related to tumor proliferation and metastasis, and play an important role in cancers progressing. A number of studies have reported the potential of miRNAs in pre-cancer prediction, diagnosis, and treatment in the future (Figure 1).

Krüppel-like factors (KLFs) are a family of transcription factors that typically contain 3 tandem zinc fingers at the $\mathrm{C}$-terminal and regulate diverse biological activities including cell proliferation, differentiation, apoptosis, and tissue development, as well as homeostatic maintenance (5). Eighteen KLFs have been identified to date. They can recognize and bind to DNA sequences with high GC expression in target genes, such as CACCC-box $(6,7)$. Owing to the diversity of their $\mathrm{N}$-terminal sequence, KLFs are able to mediate different transcriptional effects, such as transcriptional activation or inhibition $(5,8)$. Furthermore, the dysregulated expression of some KLFs has been found to be closely related to tumor-related regulation and signal transduction pathways (9).

As a member of the KLF family, KLF4 has become well known for its ability to induce cell pluripotency. Studies have also shown it to be closely related to cancer occurrence and development, which has attracted great attention as a research focus in recent years. Like other KLFs, KLF4 contains 3 tandem zinc fingers at its C-terminal, while at its $\mathrm{N}$-terminal, there is a transactivation domain and a repressor domain, which perform transcriptional activity by binding to other factors (8). KLF4 plays an important role in cellular processes including cell proliferation, differentiation, and apoptosis. For instance, in mice, a lack of KLF4 affects epidermal cell differentiation, resulting in death due to the inability to maintain a complete skin and mucosal barrier after birth (10). However, the results of studies on the role of KLF4 in cancer have been controversial. Depending on the context, KLF4 may function as a tumor suppressor or an oncogene, and it is closely related to the clinical treatment and prognosis of patients with cancer (11). In colon cancer (12), gastric cancer (GC) (13), lung cancer (14), and bladder cancer (15), for instance, KLF4 functions as tumor suppressor, whereas in osteosarcoma (16) and skin squamous cell carcinoma (17), it exhibits oncogenic activity. Li et al. found that KLF4 might be used as a beneficial biomarker for early diagnosis and prognosis of patients with lung adenocarcinoma (18). In another study, it was found that the expression level of KLF4 is a favorable diagnostic and prognostic factor for triple-negative breast cancer patients (19). KLF4 might be utilized as a favorable biomarker for early diagnosis and prognosis of tumor (20). Due to critical role of KLF4 in cancer, it is a potential therapeutic target for cancer therapy. However, there was not many studies covering this area. To date, three drugs were reported to act on KLF4. Kaur et al. showed a preclinical CD47 antibody B6H12 inhibited breast CSC proliferation and asymmetric division in triple negative breast cancer via reducing KLF4 in part by induction of miR-7 in stem cell (21). Additionally, Sijunzi decoction, a traditional Chinese medicine prescription, could up-regulate KLF4 during colorectal cancer treatment, suggesting KLF4 is a potential therapeutic target of Sijunzi decoction (22). Moreover, a potent CDK1/cyclin B inhibitor, Kenpaullone (NSC-664704), was reported to be able to inhibit the KLF4 activity and reduce the breast cancer cell motility in vitro (23).

Abnormal expression of miRNAs can be found in various cancers. Tumor-specific miRNAs are not only reliable markers for early tumor diagnosis and prognostic prediction, but are also promising therapeutic targets (24). KLF4 is closely related to cancer initiation and progression through its function as a transcription factor. However, the regulatory machinery of KLF4 in cancer is largely elusive and requires further illustration. miRNAs, as key gene regulators, have been found to adjust and control the expression of KLF4 and affect its function in cancers. This review summarizes the miRNAs which specifically targets KLF4 and their effects on outcome in different types of cancer. We hope to provide a reliable basis for subsequent researches on cancer diagnosis, treatment, and prognosis. 


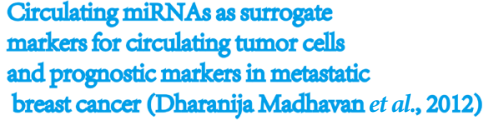

Circulating miR-22, miR-24 and miR-34a as novel predictive biomarkers in advanced non-small cell lung cancer (Tindara Franchina et al., 2014)
miRNA-1290 as a novel diagnostic and prognostic biomarker in human colorectal cancer (H Imaoka et al., 2016) Decreased circulating miRa potential biomarker for patients with non-small-cell lung cancer (Hong Yu et al., 2014)

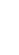

(Bs biomarker to track disease in breast cancer et al., 2012

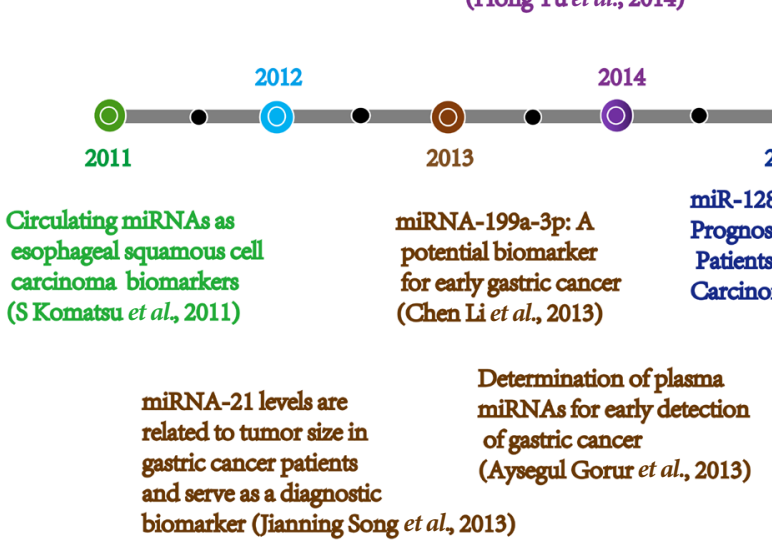

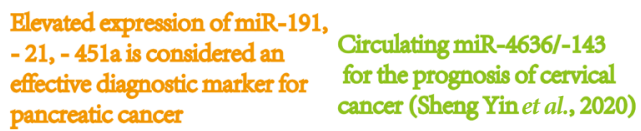

Elevated expression of miR-191, $-21,-451$ is considered an Circulating miR-4636/-143 effective diagnostic marker for for the prognosis of cervical pancreatic cancer

cancer (Sheng Yin et al., 2020)

('Takuma Goto et al., 2018)

2018

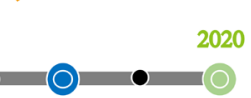

\section{7}

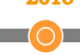
20

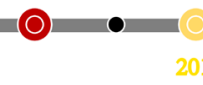

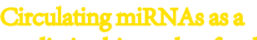
predictive biomarker for the diagnosis and recurrence of papillary thyroid carcinoma Yanging Zhang et al., 2017)
2019

miR-31-5p may be a potential diagnostic biomarker in nasopharyngeal carcinoma(S J Yiet al., 2019)

Figure 1 Timeline showing reports on microRNAs as potential biomarkers in various types of cancer.

We present the following article in accordance with the Narrative Review reporting checklist (available at http:// dx.doi.org/10.21037/atm-21-2347).

\section{Methods}

We identified research material for the current review by searching PubMed. Search terms included "miRNAs and KLF4", "KLF4 and cancer", "miRNAs and cancer", and "miRNAs, KLF4 and cancer". Based on the literatures in the last decade, the searched literatures were reviewed with the purpose of "microRNA-mediated regulation of Krüppel-like factor 4 in cancer". Relevant papers were retrieved and further results were found by reviewing related papers and the references of the retrieved papers. We then conducted a narrative overview of the literature to summarize the results of the papers.

\section{Discussion}

\section{Hepatocellular carcinoma (HCC)}

Liver cancer has become the $3^{\text {rd }}$ leading cause of cancer death in the world (25), with HCC being the most frequently diagnosed form of liver cancer. The 5 -year survival rate of patients with HCC is low, and most HCC patients are diagnosed at the advanced stage (26). Therefore, the discovery of potential biomarkers and targets for HCC therapy is critical. miRNAs constitute a novel direction in the search for potential HCC biomarkers. To date, over 300 miRNAs have been found in liver tissue, some of which are strongly related to HCC progression. In HCC, both pro-oncogenic and tumor-suppressor roles have been reported for KLF4, and further investigations are needed to determine the exact role of KLF4 in this malignancy.

$\mathrm{Wu}$ et al. reported that miR-124 was down-regulated in HCC compared to normal liver tissue, and that HCC patients with a high expression of miR-124 had a better prognosis than those with a low expression. Their results showed that miR-124 inhibited the proliferation and invasion of SMMC-7221 cells. Moreover, they demonstrated that overexpression of KLF4 enhanced the sphere formation of Hep3B cells and that miR124 reversed this outcome, indicating that KLF4 plays oncogenic role in HCC and is a functional target of miR124 (27). A study conducted by Dong et al. found that miR9-5p was overexpressed in HCC and KLF4 was downregulated, and that miR-9-5p repressed KLF4-mediated inhibition of the protein kinase B (AKT)/mammalian target of rapamycin (mTOR) signaling pathway, thereby 
promoting cell proliferation. Moreover, activation of the AKT/mTOR signaling pathway was also observed to upregulate the anti-apoptotic factors B-cell lymphoma 2 (Bcl-2) and Bcl-2-associated $\mathrm{X}$ protein (Bax), resulting in apoptosis inhibition (28). Hujie et al.'s observations, in line with those of Dong, supported the tumor suppressor role of KLF4 in HCC. Their results demonstrated that miRNA$10 \mathrm{~b}$ was up-regulated in HCC, with miRNA-10b-mediated down-regulation of KLF4 resulting in the activation of the KLF11/Smads pathway, thereby promoting the invasion of HCC cells (29). Additionally, miR-18a was reported by Liu et al. to target KLF4 and improve the motility of the HCC cell lines Hep3B and Bel-402. The study showed that miR18a targeted KLF4 to down-regulate its expression, which resulted in the up-regulation of $\mathrm{p} 21$, which is a downstream target of KLF4 (30).

\section{Colorectal cancer (CRC)}

$\mathrm{CRC}$ is one of the most common cancers, ranking $3^{\text {rd }}$ and $2^{\text {nd }}$ globally in terms of incidence and mortality, respectively, according to recent statistics (25). The pathogenesis of CRC is closely related to aging, unhealthy dietary habits, smoking, and obesity (31). Abnormal miRNA expression could be an oncogenic factor in CRC and was summarized in detail in Chen et al.'s review. While KLF4 is considered to be a tumor suppressor in CRC, several reports have shown the importance of miRNAs in the irregular expression of KLF4 in this malignancy (32). For instance, Zeng et al. showed that miR-25-3p was overexpressed in patients with CRC with metastasis, with miR-25-3p from CRC-derived exosome being a promising prognostic biomarker. Further probing showed that miR25-3p targeted KLF4 to down-regulate its downstream targets, such as zonula occludens-1 (ZO-1), occludin, and claudin-5, thereby promoting CRC metastasis (33). KLF4 was also reported to be down-regulated by miR10b in CRC by Xie et al. Similar to Zeng et al., Xie et al. showed that miR-10b was overexpressed in CRC patients with metastasis compared to those without. KLF4 was also found to be 1 of the key downstream targets of miR$10 \mathrm{~b}$, with a low expression of KLF4 inhibiting E-cadherin expression and up-regulating Snail expression, thus promoting endothelial-mesenchymal transition (EMT) and metastasis (34). An investigation conducted by Chen et al. also supported a tumor suppressor role of KLF4 in CRC. Their in silico binding and correlation analyses based on The Cancer Genome Atlas (TCGA) database showed that miR-92a was up-regulated in primary tumor tissue compared to the adjacent normal tissue and targeted KLF4. However, although they suggested that miR-92a mediated the down-regulation of KLF4, they did not provide further molecular experimental evidence to prove this thesis (35).

\section{$G C$}

GC is one of the most common malignant cancers in the world, ranking $4^{\text {th }}$ in terms of mortality (25). A detailed review which summarized previous data suggested that KLF4 functions as a tumor suppressor in GC (36). KLF4 could be regulated by miR-103 in GC. Zheng et al. showed that miR-103 was overexpressed in GC tissue and was negatively correlated with KLF4 expression. They also reported that KLF4 was targeted by miR-103 and that its overexpression could repress the enhancement of cell migration and invasion induced by miR-103 (37).

KLF4 also plays a role in the progression of GC induced by Helicobacter pylori (H. pylori) infection (38), which is a major cause of GC. One study suggested that miR-155 expression induced by $H$. pylori infection could inhibit DNA mismatch repair and stimulate mutation, resulting in GC. KLF4 was found to be 1 of miR-155's target genes, and by down-regulating KLF4 expression and promoting EMT, miR-155 promoted GC cell migration and invasion (39). Shao et al. showed that $H$. pylori induced the expression of another miRNA, miR-135b-5p, via activation of the nuclear factor-kappa $\mathrm{B}$ (NF-kB) signaling pathway. They found a putative miR-135b-5p binding site and confirmed KLF4 as a target of miR-135b-5p. Further investigation uncovered a low expression of KLF4 as being accountable for cisplatin resistance in GC cells, indicating that the miR-135b-5p/KLF4 axis plays an important role in drug resistance in GC (40).

\section{Prostate cancer}

Prostate cancer is a leading male cancer. KLF4 has been found to induce apoptosis of prostate cancer cells through binding to the promoter region of Bcl-2-interacting killer (BIK) and enhancing BIK expression. Zhang et al. found that overexpression of miR-32-5p induced by cisplatin (DDP) treatment could enhance drug resistance in prostate cancer by targeting KLF4 and inhibiting the KLF4/ BIK signaling pathway (41). Clinical research has also revealed a negative correlation of $\mathrm{miR}-148-3 \mathrm{p}$ and $\mathrm{miR}$ - 
152-3p expression with KLF4 expression (42). These 2 miRNAs were later confirmed to target KLF4, and KLF4 overexpression was able to reverse the growth inhibition provoked by miR-148-3p and miR-152-3p. Thus, miR$148-3 \mathrm{p} / \mathrm{miR}-152-3 \mathrm{p}-\mathrm{KLF} 4$ axis should be regarded as a potential target for prostate cancer therapy.

\section{Esophageal cancer (EC)}

$\mathrm{EC}$ is a highly aggressive malignancy with a poor prognosis. It can be classified into esophageal squamous cell carcinoma (ESCC) and esophageal adenocarcinoma (EAC), with the majority of EC cases diagnosed as ECSS $(43,44)$. An investigation conducted by Tian et al. involving 40 pairs of ESCC samples and normal mucus samples revealed miR$10 \mathrm{~b}$ to be up-regulated in ECSS (45). They observed that miR-10b enhanced the migration and invasion of ESCC cells. Further, KLF4 was found to inhibit ESCC metastasis and was targeted by miR-10b. The authors therefore suggested that the miR-10b/KLF4 axis could be a novel direction for future research.

\section{Lung cancer}

Lung cancer has been a focus of immense clinical concern due to its high mortality rate worldwide (46). Lung cancer can be categorized into 2 types based on the morphology: small cell lung cancer (SCLC), and non-small cell lung cancer (NSCLC) (47). The tumor suppressor role of KLF4 in lung cancer has been well characterized (48), and miRNAs targeting KLF4 have also been discovered. By analyzing 31 pairs of NSCLC tissue samples and corresponding normal lung tissues, Ding et al. showed that miR-25 was highly expressed in NSCLC and promoted the migration and invasion of NSCLC cells. They further demonstrated that miR-25 regulated the extracellular signal-regulated kinase (ERK) signaling pathway via its targeting of KLF4 (49). KLF4 was also found to be targeted by miR-3120-5p, which not only impacted on the cell cycle, but also promoted the migration and invasion of NSCLC cells. Another study further revealed that miR-3120-5p regulated cell invasiveness by targeting KLF4 (50).

Moreover, the involvement of KLF4 in drug-resistant NSCLC has also been reported. Cui et al. reported that the long non-coding RNA (lncRNA) metastasis-associated lung adenocarcinoma transcript (MALAT) repressed miR145 expression, leading to the up-regulation of KLF4 expression. Knockdown of MALAT induced the upregulation of miR-145 and a decrease in KLF4 expression, sensitizing A549 cells to DDP (51). However, this report did not provide further evidence to support the idea that KLF4 is a direct promoter of chemoresistance in NSCLC cells.

\section{Breast cancer}

Although the prognosis of patients with breast cancer is better and their survival rate is significantly higher now than in the past, breast cancer is still an important issue that plagues the health of women worldwide (52). Currently, most studies support the view that KLF4 plays a tumor suppressor role in breast cancer. Several miRNAs have been found to be involved in KLF4 regulation in breast cancer. Okuda et al. reported that KLF4 was negatively correlated with brain metastasis in patients with breast cancer. They demonstrated that KLF4 expression was repressed by miR-7 in a mouse model (53); further in vitro investigation showed miR-7 to inhibit the invasion and self-renewal abilities of cancer stem cells by targeting KLF4. Recently, a novel mechanism involving miR-1233-3p targeting KLF4 in breast cancer was discovered by $\mathrm{Lu}$ et al., who found that a circular RNA (circEHMT1) originating from euchromatic histone lysine methyltransferase 1 could target miR-1233-3p, thereby modulating KLF4 expression and resulting in the inhibition of breast cancer cell migration and invasion (54).

\section{Bladder cancer}

Bladder cancer is the $6^{\text {th }}$ most common cancer in men, and its incidence is increasing year by year. It imposes a heavy burden on both the quality of patient survival and social healthcare systems (25). The role of KLF4 in bladder cancer remains controversial. In bladder cancer, Xiao et al. reported that miR-10b was overexpressed and promoted cancer cell migration through its direct targeting of KLF4 and its induction of the down-regulation of the KLF4 downstream factor E-cadherin, thus indicating a tumor suppressor role of KLF4 in this malignancy (55). Conversely, Minami et al. reported that KLF4 was high expressed in clinical bladder cancer samples, and was one of the target genes of miR145 (56). Furthermore, Luo et al., showed that miR-139$5 \mathrm{p}$ inhibited the self-renewal ability of cancer stem cells in bladder cancer by targeting KLF4, along with NANOG, indicating that KLF4 has an oncogenic role in bladder 
cancer (57).

\section{Cervical cancer}

Cervical cancer ranks as the $4^{\text {th }}$ most common cancer among the global female population and the $7^{\text {th }}$ most common overall (25). Fortunately, it has been established that chronic viral infections caused by human papillomavirus (HPV) high-risk subtypes lead to the development of cervical cancer, and HPV vaccines have been created as an effective means of reducing the incidence of cervical cancer (58). Few studies have reported the role of KLF4 in human cervical cancer. Yang et al. analyzed clinical cervical carcinoma samples and found that KLF4 was down-regulated in cervical cancer. They observed that KLF4 overexpression inhibited the proliferation of cervical cancer cells, suggesting that KLF exerts a tumor suppressor effect in cervical cancer (59). Recently, Dai et al. discovered the differential expression of several miRNAs (with miR21 and miR-16 being overexpressed and miR-205 being down-regulated) in cervical cancer via bioinformatics data mining. Their study further showed KLF4 to be a target gene of miR-16, with a low expression of KLF4 being a poor prognostic factor in patients with cervical cancer, thus suggesting that KLF4 is a tumor suppressor in this malignancy (60).

\section{Glioblastoma}

Glioblastoma is a common type of brain tumor. Due to its high malignancy, poor survival, and lack of effective treatments, glioblastoma is one of the deadliest human malignancies (61). Several investigations have demonstrated that KLF4's function in glioblastoma is regulated by miR152. Ma et al. reported that miR-152 was down-regulated in glioblastoma samples and stem cells compared to normal brain tissues. Their mechanistic study further revealed that KLF4 was up-regulated in glioblastoma stem cells due to the low expression of miR-152, and that the miR-152/KLF4 axis inhibited the itogen-activated proteinkinase kinase $1 / 2$ or mitogen-activated proteinkinase kinase 1/2 (MEK1/2) and the phosphoinositide-3-kinase (PI3K) signal pathways by down-regulating the recombinant human galectin 3 (LGALS3) expression. This activity resulted in the inhibition of the proliferation, migration, and invasion of glioblastoma stem cells, and promoted their apoptosis (62). In line with Ma's study, recent research conducted by Gong showed that the miR-152/KLF4 axis enhanced the stemness of glioblastoma cells (63). The authors further discovered that the low expression of miR-152 was caused by activation of lncRNA X-inactive specific transcript (XIST) by steroid receptor coactivator-1 (SRC1).

\section{Laryngeal carcinoma}

Laryngeal squamous cell carcinoma is a common head and neck cancer. Current treatments include surgery, radiotherapy. and chemotherapy, but the survival rate of cancer patients is still extremely low (64). The role of KLF4 in laryngeal cancer has been largely elusive. Guo et al. demonstrated that miR-375 and miR-206 can inhibit the invasiveness of HepG2 cells by suppressing the KLF4 expression (65). Another report by Karatas et al. showed that miR-145 inhibited stemness characteristics of HepG2 cells by down-regulating KLF4, together with other stemness factors, such as SRY-Box transcription factor 2 (SOX2) and octamer-binding transcription factor 4 (Oct4) (66). However, these 2 reports failed to provide further evidence to support KLF4 being a direct target of these miRNAs, although miR-145 was reported to directly target KLF4 in bladder cancer.

\section{Conclusions}

KLF4 plays an important role in cancer initiation, progression, and metastasis. Abnormal expression of KLF4 has been reported almost in all kinds of cancers. The exact role of KLF4 in different cancers is context-dependent. Expression of KLF4 is regulated at several levels. For instance, at the transcriptional level, KLF4 is regulated by SIRT6 (67), Snail (67), SET8 (68), AR (69), DYRK2 (70), NFI-C (71), and EGFR (72). At the protein level, its stability is regulated by USP10 (73). KLF4 is also regulated by multiple miRNAs across many cancer types (Table 1).

miRNAs are highly conserved, and their abnormal expression is closely related to cancer development. In mechanistic studies of cancer, miRNAs and their downstream regulatory pathways have received increasing attention in recent years, and several miRNAs have become promising biomarkers for cancer diagnosis and prognosis. In this review, we have summarized published reports regarding miRNAs that directly target KLF4, and the effects of these miRNA/KLF4 axis on cell proliferation, migration, and invasion in different cancers. We hope our review will provide a new angle for future cancer therapy research and drug discovery. 
Table 1 MicroRNAs that regulate KLF4 in cancer

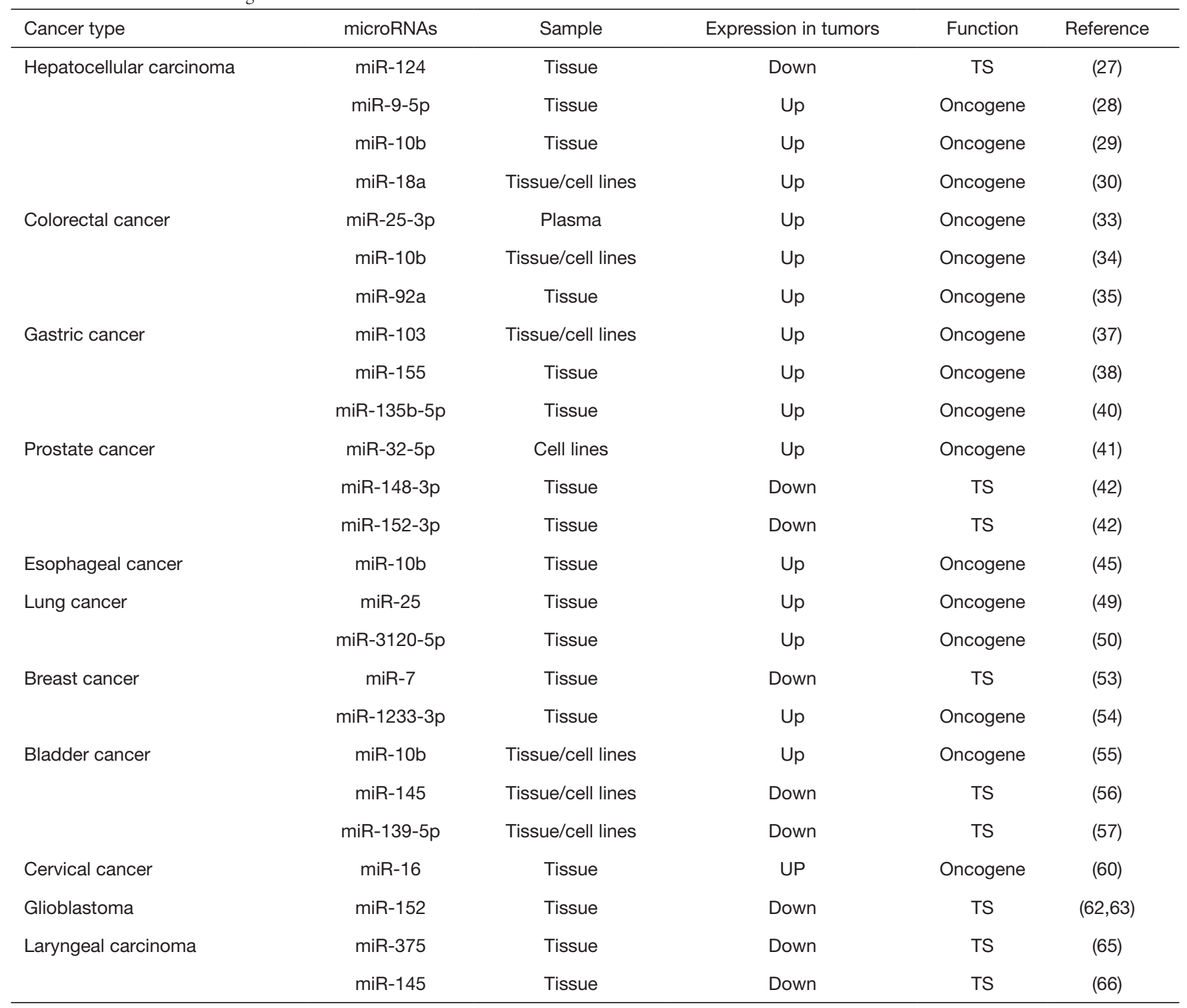

KLF4, Krüppel-like factor 4; miR, microRNA; TS, tumor suppressor; up, up-regulation; down, down-regulation.

\section{Acknowledgments}

The authors would like to thank the Instrumental Analysis Center of Shenzhen University for providing technical support.

Funding: The present study was supported by grants from the National Natural Science Foundation of China (grant No. 81802280), the Shenzhen Municipal Government of China (grant No. JCYJ20180507182427559 and GJHZ20180418190559891), the Natural Science Foundation of Guangdong Province (grant No. 2019A1515010210 and 2021A1515011046), and Shenzhen Key Medical Discipline
Construction Fund (grant No. SZXK060).

\section{Footnote}

Reporting Checklist: The authors have completed the Narrative Review reporting checklist. Available at http:// dx.doi.org/10.21037/atm-21-2347

Conflicts of Interest: All authors have completed the ICMJE uniform disclosure form (available at http://dx.doi. org/10.21037/atm-21-2347). The authors have no conflicts 
of interest to declare.

Ethical Statement: The authors are accountable for all aspects of the work in ensuring that questions related to the accuracy or integrity of any part of the work are appropriately investigated and resolved.

Open Access Statement: This is an Open Access article distributed in accordance with the Creative Commons Attribution-NonCommercial-NoDerivs 4.0 International License (CC BY-NC-ND 4.0), which permits the noncommercial replication and distribution of the article with the strict proviso that no changes or edits are made and the original work is properly cited (including links to both the formal publication through the relevant DOI and the license). See: https://creativecommons.org/licenses/by-nc-nd/4.0/.

\section{References}

1. Wang JK, Wang Z, Li G, et al. MicroRNA-125 in immunity and cancer. Cancer Lett 2019;454:134-45.

2. Lin YH. MicroRNA Networks Modulate Oxidative Stress in Cancer. Int J Mol Sci 2019. [Epub ahead of print] doi:10.3390/ijms20184497.

3. Vychytilova-Faltejskova P, Slaby O, et al. MicroRNA-215: From biology to theranostic applications. Mol Aspects Med 2019;70:72-89.

4. Hosseinahli N, Aghapour M, Duijf PHG, et al. Treating cancer with microRNA replacement therapy: A literature review. J Cell Physiol 2018;233:5574-88.

5. Oishi Y, Manabe I, et al. Krüppel-Like Factors in Metabolic Homeostasis and Cardiometabolic Disease. Front Cardiovasc Med 2018;5:69.

6. Guan F, Kang Z, Zhang JT, et al. KLF7 promotes polyamine biosynthesis and glioma development through transcriptionally activating ASL. Biochem Biophys Res Commun 2019;514:51-7.

7. Pearson R, Fleetwood J, Eaton S, et al. Krüppel-like transcription factors: a functional family. Int $\mathrm{J}$ Biochem Cell Biol 2008;40:1996-2001.

8. Rane MJ, Zhao Y, Cai L, et al. Krüppel-like factors (KLFs) in renal physiology and disease. EBioMedicine 2019;40:743-50.

9. Zhang J, Li G, Feng L, et al. Krüppel-like factors in breast cancer: Function, regulation and clinical relevance. Biomed Pharmacother 2020;123:109778.

10. Dang DT, Mahatan CS, Dang LH, et al. Expression of the gut-enriched Krüppel-like factor (Krüppel-like factor 4) gene in the human colon cancer cell line RKO is dependent on CDX2. Oncogene 2001;20:4884-90.

11. Cheng Z, Zou X, Jin Y, et al. The Role of KLF4 in Alzheimer's Disease. Front Cell Neurosci 2018;12:325.

12. Rong Z, Luo Z, Zhang J, et al. GINS complex subunit 4, a prognostic biomarker and reversely mediated by Krüppellike factor 4, promotes the growth of colorectal cancer. Cancer Sci 2020;111:1203-17.

13. Zhang J, Zhu Z, Wu H, et al. PODXL, negatively regulated by KLF4, promotes the EMT and metastasis and serves as a novel prognostic indicator of gastric cancer. Gastric Cancer 2019;22:48-59.

14. Jia Y, Ying X, Zhou J, et al. The novel KLF4/PLAC8 signaling pathway regulates lung cancer growth. Cell Death Dis 2018;9:603.

15. Xie H, Li J, Ying Y, et al. METTL3/YTHDF2 m6 A axis promotes tumorigenesis by degrading SETD7 and KLF4 mRNAs in bladder cancer. J Cell Mol Med 2020;24:4092-104.

16. Qi XT, Li YL, Zhang YQ, et al. KLF4 functions as an oncogene in promoting cancer stem cell-like characteristics in osteosarcoma cells. Acta Pharmacol Sin 2019;40:546-55.

17. Chen YJ, Wu CY, Chang CC, et al. Nuclear Krüppel-like factor 4 expression is associated with human skin squamous cell carcinoma progression and metastasis. Cancer Biol Ther 2008;7:777-82.

18. Li S, Huang L, Gu J, et al. Restoration of KLF4 Inhibits Invasion and Metastases of Lung Adenocarcinoma through Suppressing MMP2. J Cancer 2017;8:3480-9.

19. Nagata T, Shimada $Y$, Sekine $S$, et al. KLF4 and NANOG are prognostic biomarkers for triple-negative breast cancer. Breast Cancer 2017;24:326-35.

20. Valencia-Hip lito A, Hernández-Atenógenes $M$, Vega GG, et al. Expression of KLF4 is a predictive marker for survival in pediatric Burkitt lymphoma. Leuk Lymphoma 2014;55:1806-14.

21. Kaur S, Elkahloun AG, Singh SP, et al. A functionblocking CD47 antibody suppresses stem cell and EGF signaling in triple-negative breast cancer. Oncotarget 2016;7:10133-52.

22. Jie $Y, H e W$, Yang $X$, et al. Krüppel-like factor 4 acts as a potential therapeutic target of Sijunzi decoction for treatment of colorectal cancer. Cancer Gene Ther 2017;24:361-6.

23. Shi L, Tang X, Qian M, et al. A SIRT1-centered circuitry regulates breast cancer stemness and metastasis. Oncogene 2018;37:6299-315.

24. Wang $Z$, Sha HH, Li HJ, et al. Functions and mechanisms 
of miR-186 in human cancer. Biomed Pharmacother 2019;119:109428.

25. Sung H, Ferlay J, Siegel RL, et al. Global Cancer Statistics 2020: GLOBOCAN Estimates of Incidence and Mortality Worldwide for 36 Cancers in 185 Countries. CA Cancer J Clin 2021;71:209-49.

26. Wei Y, Chen X, Liang C, et al. A Noncoding Regulatory RNAs Network Driven by Circ-CDYL Acts Specifically in the Early Stages Hepatocellular Carcinoma. Hepatology 2020;71:130-47.

27. Wu LP, Wu J, Shang A, et al. miR-124 inhibits progression of hepatocarcinoma by targeting KLF4 and promises a novel diagnostic marker. Artif Cells Nanomed Biotechnol 2018;46:159-67.

28. Dong X, Wang F, Xue Y, et al. MicroRNA-9-5p downregulates Klf4 and influences the progression of hepatocellular carcinoma via the AKT signaling pathway. Int J Mol Med 2019;43:1417-29.

29. Hujie G, Zhou SH, Zhang H, et al. MicroRNA-10b regulates epithelial-mesenchymal transition by modulating KLF4/KLF11/Smads in hepatocellular carcinoma. Cancer Cell Int 2018;18:10.

30. Liu L, Cai X, Liu E, et al. MicroRNA-18a promotes proliferation and metastasis in hepatocellular carcinoma via targeting KLF4. Oncotarget 2017;8:68263-9.

31. Dekker E, Tanis PJ, Vleugels JLA, et al. Colorectal cancer. Lancet 2019;394:1467-80.

32. Chen B, Xia Z, Deng YN, et al. Emerging microRNA biomarkers for colorectal cancer diagnosis and prognosis. Open Biol 2019;9:180212.

33. Zeng Z, Li Y, Pan Y, et al. Cancer-derived exosomal miR-25-3p promotes pre-metastatic niche formation by inducing vascular permeability and angiogenesis. Nat Commun 2018;9:5395.

34. Xie Y, Zhao J, Liang Y, et al. MicroRNA-10b controls the metastasis and proliferation of colorectal cancer cells by regulating Krüppel-like factor 4. Artif Cells Nanomed Biotechnol 2019;47:1722-9.

35. Chen E, Li Q, Wang H, et al. MiR-92a promotes tumorigenesis of colorectal cancer, a transcriptomic and functional based study. Biomed Pharmacother 2018;106:1370-7.

36. Hashimoto I, Nagata T, Sekine S, et al. Prognostic significance of KLF4 expression in gastric cancer. Oncol Lett 2017;13:819-26.

37. Zheng J, Liu Y, Qiao Y, et al. miR-103 Promotes Proliferation and Metastasis by Targeting KLF4 in Gastric Cancer. Int J Mol Sci 2017. [Epub ahead of print] doi:10.3390/ijms18050910.

38. Zhao R, Liu Z, Xu W, et al. Helicobacter pylori infection leads to KLF4 inactivation in gastric cancer through a TET1-mediated DNA methylation mechanism. Cancer Med 2020;9:2551-63.

39. Ou Y, Ren H, Zhao R, et al. Helicobacter pylori CagA promotes the malignant transformation of gastric mucosal epithelial cells through the dysregulation of the miR-155/ KLF4 signaling pathway. Mol Carcinog 2019;58:1427-37.

40. Shao L, Chen Z, Soutto M, et al. Helicobacter pyloriinduced miR-135b-5p promotes cisplatin resistance in gastric cancer. FASEB J 2019;33:264-74.

41. Zhang L, Li X, Chao Y, et al. KLF4, a miR-32-5p targeted gene, promotes cisplatin-induced apoptosis by upregulating BIK expression in prostate cancer. Cell Commun Signal 2018;16:53.

42. Feng F, Liu H, Chen A, et al. miR-148-3p and miR-1523 p synergistically regulate prostate cancer progression via repressing KLF4. J Cell Biochem 2019;120:17228-39.

43. Minashi K, Nihei K, Mizusawa J, et al. Efficacy of Endoscopic Resection and Selective Chemoradiotherapy for Stage I Esophageal Squamous Cell Carcinoma. Gastroenterology 2019;157:382-390.e3.

44. Kashyap MK, Abdel-Rahman O, et al. Expression, regulation and targeting of receptor tyrosine kinases in esophageal squamous cell carcinoma. Mol Cancer 2018;17:54.

45. Tian Y, Luo A, Cai Y, et al. MicroRNA-10b promotes migration and invasion through KLF4 in human esophageal cancer cell lines. J Biol Chem 2010;285:7986-94.

46. Herbst RS, Morgensztern D, Boshoff C, et al. The biology and management of non-small cell lung cancer. Nature 2018;553:446-54

47. Duruisseaux M, Esteller M, et al. Lung cancer epigenetics: From knowledge to applications. Semin Cancer Biol 2018;51:116-28.

48. Zhou Y, Hofstetter WL, He Y, et al. KLF4 inhibition of lung cancer cell invasion by suppression of SPARC expression. Cancer Biol Ther 2010;9:507-13.

49. Ding X, Zhong T, Jiang L, et al. miR-25 enhances cell migration and invasion in non-small-cell lung cancer cells via ERK signaling pathway by inhibiting KLF4. Mol Med Rep 2018;17:7005-16.

50. $\mathrm{Xu} \mathrm{H}$, Wen Q, et al. miR-3120-5p acts as a diagnostic biomarker in non-small cell lung cancer and promotes cancer cell proliferation and invasion by targeting KLF4. Mol Med Rep 2018;18:4621-8.

51. Cui Y, Li G, Zhang X, et al. Increased MALAT1 
expression contributes to cisplatin resistance in non-small cell lung cancer. Oncol Lett 2018;16:4821-8.

52. Thorat MA, Balasubramanian R, et al. Breast cancer prevention in high-risk women. Best Pract Res Clin Obstet Gynaecol 2020;65:18-31.

53. Okuda H, Xing F, Pandey PR, et al. miR-7 suppresses brain metastasis of breast cancer stem-like cells by modulating KLF4. Cancer Res 2013;73:1434-44.

54. Lu M, Wu Y, Zeng B, et al. CircEHMT1 inhibits metastatic potential of breast cancer cells by modulating miR-1233-3p/KLF4/MMP2 axis. Biochem Biophys Res Commun 2020;526:306-13.

55. Xiao H, Li H, Yu G, et al. MicroRNA-10b promotes migration and invasion through KLF4 and HOXD10 in human bladder cancer. Oncol Rep 2014;31:1832-8.

56. Minami K, Taniguchi K, Sugito N, et al. MiR-145 negatively regulates Warburg effect by silencing KLF4 and PTBP1 in bladder cancer cells. Oncotarget 2017;8:33064-77.

57. Luo H, Yang R, Li C, et al. MicroRNA-139-5p inhibits bladder cancer proliferation and self-renewal by targeting the Bmil oncogene. Tumour Biol 2017;39:1010428317718414.

58. Lei J, Ploner A, Elfström KM, et al. HPV Vaccination and the Risk of Invasive Cervical Cancer. N Engl J Med 2020;383:1340-8.

59. Yang WT, Zheng PS, et al. Krüppel-like factor 4 functions as a tumor suppressor in cervical carcinoma. Cancer 2012;118:3691-702.

60. Dai F, Chen G, Wang Y, et al. Identification of candidate biomarkers correlated with the diagnosis and prognosis of cervical cancer via integrated bioinformatics analysis. Onco Targets Ther 2019;12:4517-32.

61. Tan AC, Ashley DM, López GY, et al. Management of glioblastoma: State of the art and future directions. CA Cancer J Clin 2020;70:299-312.

62. Ma J, Yao Y, Wang P, et al. MiR-152 functions as a tumor suppressor in glioblastoma stem cells by targeting Krüppel-like factor 4. Cancer Lett 2014;355:85-95.

63. Gong $M$, Wang X, Mu L, et al. Steroid receptor

Cite this article as: Yang X, Chen C, Li L, Xiao T, Zou YD, Zheng D. Current research advances in microRNA-mediated regulation of Krüppel-like factor 4 in cancer: a narrative review. Ann Transl Med 2021;9(11):948. doi: 10.21037/atm-21-2347 coactivator-1 enhances the stemness of glioblastoma by activating long noncoding RNA XIST/miR-152/KLF4 pathway. Cancer Sci 2021;112:604-18.

64. Li W, Chen Y, Nie X, et al. Regulatory Mechanisms of lncRNAs and Their Target Gene Signaling Pathways in Laryngeal Squamous Cell Carcinoma. Front Pharmacol 2020;11:1140.

65. Guo Y, An R, Zhao R, et al. miR-375 exhibits a more effective tumor-suppressor function in laryngeal squamous carcinoma cells by regulating KLF4 expression compared with simple co-transfection of miR-375 and miR-206. Oncol Rep 2016;36:952-60.

66. Karatas OF, Suer I, Yuceturk B, et al. The role of miR-145 in stem cell characteristics of human laryngeal squamous cell carcinoma Hep-2 cells. Tumour Biol 2016;37:4183-92.

67. Li Z, Huang J, Shen S, et al. SIRT6 drives epithelial-tomesenchymal transition and metastasis in non-small cell lung cancer via snail-dependent transrepression of KLF4. J Exp Clin Cancer Res 2018;37:323.

68. Chen X, Ding X, Wu Q, et al. Monomethyltransferase SET8 facilitates hepatocellular carcinoma growth by enhancing aerobic glycolysis. Cell Death Dis 2019;10:312.

69. Siu MK, Suau F, Chen WY, et al. KLF4 functions as an activator of the androgen receptor through reciprocal feedback. Oncogenesis 2016;5:e282.

70. Mimoto R, Imawari Y, Hirooka S, et al. Impairment of DYRK2 augments stem-like traits by promoting KLF4 expression in breast cancer. Oncogene 2017;36:1862-72.

71. Lee HK, Lee DS, Park JC, et al. Nuclear factor I-C regulates $\mathrm{E}$-cadherin via control of $\mathrm{KLF} 4$ in breast cancer. BMC Cancer 2015;15:113.

72. Pang L, Xu L, Yuan C, et al. Activation of EGFR-KLF4 positive feedback loop results in acquired resistance to sorafenib in hepatocellular carcinoma. Mol Carcinog 2019;58:2118-26.

73. Wang X, Xia S, Li H, et al. The deubiquitinase USP10 regulates KLF4 stability and suppresses lung tumorigenesis. Cell Death Differ 2020;27:1747-64.

(English Language Editor: J. Reynolds) 